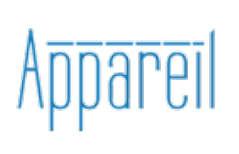

Appareil

11 | 2013

L'espace et l'architecture: état des lieux

\title{
Mur-rideau ; la façade en jeu
}

\section{Sandrine Amy}

\section{(2) OpenEdition}

Journals

Édition électronique

URL : http://journals.openedition.org/appareil/1761

DOI : 10.4000/appareil. 1761

ISSN : 2101-0714

Éditeur

MSH Paris Nord

\section{Référence électronique}

Sandrine Amy, « Mur-rideau ; la façade en jeu », Appareil [En ligne], 11 | 2013, mis en ligne le 10 juin 2013, consulté le 30 juillet 2020. URL : http://journals.openedition.org/appareil/1761 ; DOI : https:// doi.org/10.4000/appareil.1761

Ce document a été généré automatiquement le 30 juillet 2020.

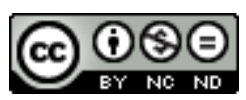

Appareil est mis à disposition selon les termes de la Licence Creative Commons Attribution - Pas d'Utilisation Commerciale - Pas de Modification 4.0 International. 


\section{Mur-rideau ; la façade en jeu}

\section{Sandrine Amy}

Dans son ouvrage Paris, capitale du XIX $x^{e}$ siècle, Walter Benjamin cite Sigfried Giedion :

Chaque époque ou presque semble, de par sa constitution interne, développer tout particulièrement un problème architectural précis : pour le gothique, ce sont les cathédrales, pour le baroque le château et pour le XIX ${ }^{e}$ siècle naissant, qui a tendance à se tourner en arrière et à se laisser ainsi imprégner par le passé, le musée.

\section{Il ajoute :}

Mon analyse trouve son objet principal dans cette soif du passé et fait apparaître l'intérieur du musée comme un intérieur élevé à une puissance considérable ${ }^{1}$.

3 Les passages parisiens partagent selon lui avec les musées ce même intérieur sans extérieur, cette même intériorité "considérable », ils sont, les uns et les autres, "des maisons ou des corridors qui n'ont pas de côté extérieur ${ }^{2}$ ", ce qui, selon Benjamin, permet de les comparer à des rêves ; musées et passages sont des « maison[s] de rêve du collectif ».

4 Avec ces deux "problèmes » architecturaux, il ouvre donc le chantier d'un rapport direct entre espace et temps, et plus précisément entre espace et histoire.

Pour l'individu comme pour le collectif, le propre de chaque rêve est de voir lui succéder le réveil, c'est un phénomène historique.

Le réveil comme processus graduel [...] s'impose dans la vie de l'individu comme dans celle des générations. Le sommeil est le stade primaire de celles-ci. L'expérience de la jeunesse pour une génération a beaucoup de points communs avec l'expérience du rêve. Sa figure historique est une figure de rêve ${ }^{3}$.

6 «Nous devons [dit-il] nous réveiller de ce qui fut l'existence de nos parents ${ }^{4}$ » pour pouvoir, à notre tour, rêver notre existence propre mais de ce rêve, il faut aussi se réveiller pour exister. "Chaque époque, en effet, ne rêve pas seulement de la prochaine et cherche au contraire dans son rêve à s'arracher au sommeil ${ }^{5}$ ».

7 La qualification de l'architecture des passages comme " maison de rêve " l'inscrit donc d'emblée dans un devenir historique dont le réveil est l'horizon. 
8 La tâche que Benjamin se donne, c'est de dégager cet instant du réveil, ce qu'il appelle « le Maintenant de la connaissabilité », l'enjeu consistant à pointer le moment de la relève d'une époque - en l'occurrence le XIX siècle - et à identifier un possible enchaînement.

9 C'est, semble-t-il, dans l'architecture moderne, et particulièrement chez Le Corbusier, qu'il avait trouvé les preuves que son époque (le $\mathrm{xx}^{\mathrm{e}}$ siècle) cherchait à s'arracher au sommeil et à se libérer de celle qui l'avait précédée.

10 Cependant, la réponse à la question de savoir si l'architecture moderne constituait les signes d'un véritable réveil nous paraît pourtant, à la lumière de ses différents textes évoquant l'architecture, problématique.

11 Benjamin nous semble en effet en faire une lecture assez ambivalente et être partagé entre l'enthousiasme et la déception.

D’un côté, par les yeux de Giedion, Benjamin, dans Paris, capitale du XIX ${ }^{e}$ siècle, voit Le Corbusier de manière positive.

L'architecture moderne se défait des masques historisants que le XIX ${ }^{\mathrm{e}}$ siècle a revêtu, de la pierre sur le fer, du stuc sur la structure - elle se détache clairement de la tradition décorative - pour se tourner, sans aucune nostalgie, vers un langage nouveau, celui de l'ingénieur.

14 Comme l'indique Giedion, avec les progrès techniques, « une fatale monumentalité de caractère patrimonial » n'a plus aucune raison d'être ;

tant que le jeu de la poussée et du support tirait son sens des murs porteurs, réellement ou avec une exagération symbolique (le baroque), la pesanteur avait une justification. Mais aujourd'hui, alors que le mur extérieur n'a plus à supporter la poussée, le jeu de la poussée et du support, souligné par l'ornement, est une pénible farce (les gratte-ciel américains) ${ }^{6}$.

D'un autre côté cependant, si Benjamin considère l'architecture moderne comme salutaire - voire vitale-, il ne peut s'empêcher de l'envisager aussi comme une irréparable perte.

Il écrit dans Expérience et pauvreté :

le cours de l'expérience a chuté, et ce dans une génération qui fit en 1914-1918 l'une des expériences les plus effroyables de l'histoire universelle. [...] cette dévalorisation n'avait rien d'étonnant. Car jamais expériences acquises n'ont été aussi radicalement démenties [...]. Cet effroyable déploiement de la technique plongea les hommes dans une pauvreté tout à fait nouvelle [et] cette pauvreté ne porte pas seulement sur nos expériences privées, mais aussi sur les expériences de l'humanité tout entière. Et c'est donc une nouvelle espèce de barbarie ${ }^{7}$.

17 Mais selon lui, cette barbarie possède un versant positif ; " car à quoi sa pauvreté en expérience amène-t-elle le barbare ? Elle l'amène à recommencer au début, à reprendre à zéro, à se débrouiller avec peu, à construire avec presque rien, sans tourner la tête de droite ni de gauche. »

18 À condition de faire table rase du passé et de la tradition, il est possible selon lui d'envisager d'aller de l'avant et de construire à nouveau. Parmi d'autres "grands créateurs » ou « constructeurs » ayant cette capacité, il cite l'architecte Adolf Loos qui repoussait «l'image traditionnelle, noble, solennelle, d'un homme paré de toutes les offrandes sacrificatoires du passé, pour se tourner vers leur contemporain qui, 
dépouillé de ces oripeaux, crie comme un nouveau-né dans les langes sales de cette époque ${ }^{8}$.»

Pour réaliser ce projet moderne, écrit-il, Le Corbusier et Loos auront recours au verre. «Le verre, ce n'est pas un hasard, est un matériau dur et lisse sur lequel rien n'a de prise. Un matériau froid et sobre, également. Les objets de verre n'ont pas d'“aura". Le verre, d'une manière générale, est l'ennemi du mystère. Il est aussi l'ennemi de la propriété9 ".

De la même manière,

Vivre dans une maison de verre est, par excellence, une vertu révolutionnaire. Cela aussi est une ivresse, un exhibitionnisme moral dont nous avons grand besoin. La discrétion sur ses affaires privées, jadis vertu aristocratique, est devenue de plus en plus le fait de petits-bourgeois arrivés ${ }^{10}$.

Mais d'un autre côté, dans son texte Le caractère destructif, il écrit en 1931 :

Le caractère destructif ne connaît qu'un mot d'ordre : faire de la place; une seule activité : déblayer. Son besoin d'air frais et d'espace libre est plus fort que toute haine. [...] Le caractère destructif n'a pas d'images en tête. Il a peu de besoins et surtout pas celui de savoir ce qui viendra à la place de ce qui est détruit. [...] Le caractère destructif fait son travail, il esquive seulement la création. [...] Le caractère destructif est l'ennemi de l'homme-étui. L'homme-étui cherche son confort et sa coquille est sa quintessence. L'intérieur de la coquille est la trace tapissée de velours qu'il a imprimée sur le monde. Le caractère destructif efface même les traces de la destruction ${ }^{11}$.

Comme le souligne Vicente Valero dans son ouvrage Expérience et pauvreté, Walter Benjamin à Ibiza, ce sentiment d'ambivalence est sans doute particulièrement exacerbé chez Benjamin du fait qu'il séjourne, lors de l'écriture de Expérience et pauvreté en 1933, dans l'île. C'est en tout cas dans une sorte de temps suspendu « entre la guerre de 1914 et celle qui était sur le point d'éclater » et dans un lieu - une île - "pas encore touché par la force irrésistible du capitalisme [et de l'architecture industrielle], comme un monde retiré du monde ${ }^{12}$ » qu'il était sans doute le mieux à même de mesurer l'écart entre l'architecture vernaculaire et l'architecture de son temps.

L'habitat rural ibizien - qui ne connaissait ni le verre ni l'acier, bien évidemment suggéra à Benjamin, [...] quelques réflexions sur la modernité et, plus particulièrement, sur les pertes que celle-ci entraînait inévitablement ${ }^{13}$.

Dans De l'espace pour ce qui est précieux, Benjamin écrit à propos d'Ibiza ;

Dans la maison où il n'y a pas de lit est précieux le tapis avec lequel l'habitant se couvre la nuit, dans la voiture qui n'a pas de rembourrage le coussin que l'on pose sur son dur châssis. Mais dans nos maisons bien équipées il n'y a pas d'espace pour ce qui est précieux, parce qu'il n'y a pas de marge de manœuvre pour ses services ${ }^{14}$.

Ailleurs il confirme :

D'habitants des maisons, on veut nous transformer en usagers, de fiers propriétaires en dédaigneux utilisateurs ${ }^{15}$.

Le prix à payer pour entrer dans la modernité combinait donc à la fois la perte de l'aura mais aussi la perte d'humanité, car il concernait tout autant les objets que les individus ; "Pauvres, voilà bien ce que nous sommes devenus. Pièce par pièce, nous avons dispersé l'héritage de l'humanité, nous avons dû laisser ce trésor au mont-depiété, souvent pour un centième de sa valeur, en échange de la piécette de l'« actuel ${ }^{16}$ ".

En tentant de libérer l'habitant de la tyrannie de "son cadre domestique »-c'est-àdire du «salon bourgeois des années 1880 » qui excluait, tant il était surchargé de 
bibelots, toute présence étrangère et rendait esclave son propriétaire - pour privilégier "le souci de sa propre personne ", l'architecture prenait donc nécessairement le risque de déshumaniser aussi bien la maison que son occupant.

Cependant, on peut penser que, dans l'esprit de Benjamin, la perte de l'aura et de l'humanité n'était pas plus destinée à perdurer que la force - énergique, barbare ou révolutionnaire - qui les avait engendrées et que du prix à payer pour que son époque entre dans la modernité, le monde ne devait s'acquitter qu'une seule fois.

Cette époque posait semble-t-il deux impératifs pour Benjamin ; il s'agissait d'une part de sortir du cauchemar de la guerre qui avait eu lieu, mais il s'agissait, d'autre part, de sortir du rêve du XIXe siècle dont, du fait même de cette guerre, il n'avait pas été possible de se réveiller.

L'urgence d'un renversement dialectique, d'un « renversement révolutionnaire » ou d'une "révolution copernicienne ", c'est-à-dire politique, devait donc se faire sentir à double titre afin, non pas de faire se terminer l'histoire, mais de la prolonger en rétablissant l'enchaînement alternatif sommeil/réveil que le cauchemar de la première guerre mondiale avait vraisemblablement interrompu.

On peut donc postuler que la barbarie positive n'était qu'un moyen, tout à fait temporaire, au service du rétablissement de l'histoire, le rêve devant finir un jour par remplacer le cauchemar, le réveil le sommeil et l'expérience reprendre, même renouvelée, son « cours » normal.

Rétrospectivement, et contrairement à ce qu'en disait Benjamin à l'époque, il semble que les architectes modernes ont usé de la barbarie d'une manière ni tout à fait " positive » ni tout à fait provisoire ou, ce qui revient au même, que la barbarie qui était la leur a été d'une telle violence que sa force d'inertie s'est propagée à leurs descendants, est parvenue jusqu'à nous et a empêché, à de nombreux égards, qu'un véritable réveil et qu'une véritable relève de l'architecture du XIX ${ }^{e}$ siècle, du monde de la structure et de l'intériorité n'aient lieu.

Si l'on suit en effet Benjamin,

On ne peut véritablement se délivrer d'une époque que par un geste qui a la structure du réveil en ceci également qu'il est entièrement régi par la ruse. C'est par la ruse, et elle seule, que nous nous arrachons au royaume du rêve. Mais il y a aussi une fausse délivrance dont la violence est le signe. Elle a condamné dès le début le modern style au déclin ${ }^{17}$.

Aux côtés des architectes du mouvement moderne, il présente le modern style comme le représentant de cette violence et de cet "effort infructueux ${ }^{18}$ " pour s'arracher au sommeil - il dit dans Le livre des passages : « Le modern style, c'est rêver qu'on est éveillé 19 »- mais avec la distance critique, il nous semble que l'architecture moderne a révélé qu'elle projetait plutôt là une violence et une incapacité dont elle était elle-même habitée.

Citons les deux aspects les plus frappants de cette violence.

L'utilisation généralisée du blanc d'abord (le blanc comme uniforme) et l'éradication de la couleur et du décor. Pour les modernes en effet,

La couleur libre, livrée à elle-même, est le grand danger, la menace que doit anticiper l'architecture en l'accueillant pour la soumettre et lui permettre de jouer son rôle de faire-valoir du blanc, émousser sa pointe acérée, calmer son rythme effréné qui mettrait en valeur son tropisme décoratif pour la reterritorialiser à titre de valeur déterminée, soumise, conformément à son destin d'ornement. «Quantum 
du paysan » (Le Corbusier), la couleur dessine ainsi trois périphéries : sociale - elle connote alors un goût populaire, tapageur; sexuelle-elle échoue à la femme à travers le fard ; géographique - la couleur est l'exotisme ${ }^{20}$.

L'impératif de transparence ensuite. Giedion constate que :

Les maisons de Corbusier ne sont ni spatiales, ni plastiques : l'air les traverse ! L'air devient facteur constitutif ! Il s'agit non plus d'espace ou de plastique, mais bien de RELATION et de PÉNÉTRATION. Il n'existe plus qu'un seul espace indivisible. Entre intérieur et extérieur les séparations sont tombées ${ }^{21}$.

Mais il n'ignore pas qu'il y a là un danger ;

Il semble qu'il y ait là une limite à ne pas dépasser. [...] Question : habitués que nous sommes à marcher d'un pas pressé, avons-nous assez de force intérieure pour arpenter une de ces terrasses que baigne l'air comme des projecteurs baignent de lumière une scène de théâtre, sans avoir le sentiment d'être sur cette scène ? - La génération montante d'architectes sent bien que, là, il y a danger ${ }^{22}$.

Comme le souligne aussi Benjamin, le problème de l'architecture de verre et des «formes techniques de construction (par opposition aux formes artistiques) ont ceci de spécifique que leur progrès et leur réussite sont proportionnels à la transparence de leur contenu social ${ }^{23} »$.

Contrairement à Benjamin, il nous semble donc que Le Corbusier ne constitue pas le réveil tant espéré mais plutôt une sorte de "fausse délivrance » du passé et de la tradition.

Cette hypothèse nous semble pouvoir être confirmée par le fait que plusieurs architectes, à la même époque, montrent les mêmes signes de violence, faisant prendre à cette fausse délivrance, indépendamment de ses diverses manifestations, un caractère collectif.

41 Si l'on considère le travail de Loos - largement passé sous silence par Giedion mais comportant de nombreuses affinités avec celui de Le Corbusier - qui, lui aussi, prône la disparition du décor et qualifie de criminel tout recours à l'ornement, il apparaît en effet qu'au moment même où la nature totalitaire de la transparence de Le Corbusier effleure l'esprit de Giedion, se construit celle, symétrique, de l'opacité.

Pour l'un et l'autre, c'est comme si la façade n'avait que pour seule urgence de libérer une violence latente (Le Corbusier) ou de la contenir (Loos), et pour seule fin, d'être à son tour infligée, projetée au-dehors dans la perspective de détruire tout secret (Le Corbusier) ou de le sceller par tous les moyens (Loos).

3 Si on peut effectivement dire des architectures de Le Corbusier qu'elles empêchaient toute trace; de celles de Loos, on peut dire qu'elles procuraient encore à leurs occupants l'étui douillet bourgeois du XIX ${ }^{\mathrm{e}}$ siècle, à condition qu'ils se laissent emmurer derrière des façades quasiment hermétiques à toute vie publique.

44 Pour l'un et l'autre, nous semblons bien être confrontés à la même loi qui, selon Benjamin, veut que dans la fausse libération, "tout effort produise son contraire ${ }^{24}$ ". Sous couvert de vouloir émanciper et libérer l'homme moderne, l'architecture de l'époque a semblé vouloir en finir avec l'habitat et l'expérience même de l'habiter, menaçant du même coup l'habitant dans son individualité et risquant finalement de l'asservir.

45 Et malgré leurs efforts, aucun des deux n'a pu se débarrasser du décor - le blanc reste une décoration, aussi minimale soit-elle - mais, au contraire, ils en ont réaffirmé la nécessité et l'inamovibilité. Aucun des deux n'a pu non plus faire l'économie de la 
façade - en la faisant disparaitre ou en la compassant - pour la soumettre à la seule nécessité ou à la seule structure interne, mais, au contraire, ils ont réaffirmé la dimension éminemment vitale de sa fonction d'interface.

Cependant, si elle l'a retardée, cette violence n'a pas empêché les architectes modernes de faire un nouveau rêve. Comme l'écrit Benjamin :

La pauvreté en expérience: cela ne signifie pas que les hommes aspirent à une expérience nouvelle. Non, ils aspirent à se libérer de toute expérience quelle qu'elle soit, ils aspirent à un environnement dans lequel ils puissent faire valoir leur pauvreté, extérieure et finalement aussi intérieure, à l'affirmer si clairement et si nettement qu'il en sorte quelque chose de valable. Ils ne sont du reste pas toujours ignorants ou inexpérimentés. On peut souvent dire le contraire: ils ont « ingurgité » tout cela, la « culture » et l'« homme », ils en sont dégoûtés et fatigués. [...] À la fatigue succède le sommeil, et il n'est alors pas rare que le rêve nous dédommage de la tristesse et du découragement de la journée, en réalisant l'existence très simple, mais vraiment grandiose, que nous n'avons pas la force de construire dans l'état de veille ${ }^{25}$.

C'est pourquoi pour lui, si chaque époque a un côté tourné vers la veille, qui est son côté adulte, elle conserve toujours un autre "côté tourné vers des rêves, qui est son côté enfantin ${ }^{26} »$.

C'est, on peut en faire l'hypothèse, ce que Benjamin avait vu ou pressenti dans l'œuvre de Le Corbusier; cette fausse délivrance masquait malgré tout une ruse qui le concernait directement et était susceptible, elle, d'annoncer un réveil proche.

Effet miroir en effet; c'est au moment où lui-même, dans la veille, proposait une interprétation du rêve du $\mathrm{XIX}^{\mathrm{e}}$ siècle (depuis le $\mathrm{Xx}^{\mathrm{e}}$ ) qu'il était déjà lui-même, avec d'autres, en train de rêver le suivant, c'est-à-dire le xxI siècle.

On peut en effet supposer que si Benjamin travaillait sur les passages et était en mesure de les qualifier de "rêves", c'est que non seulement il avait acquis la distance nécessaire pour le faire - c'est-à-dire qu'il était lui-même réveillé de ce rêve là - mais aussi qu'il en avait lui-même commencé un autre, le sien propre, qui lui apparaissait déjà par bribes et dont il essayait de se réveiller.

51 Ainsi, ne pouvait-il pas ne pas remarquer que tandis que, d'un côté, les architectes tels que Loos et Le Corbusier construisaient leurs édifices selon les lois rigoureuses du mouvement moderne (lois qu'ils avaient eux-mêmes édictées et lois du marché et de l'industrie de leurs ainés auxquelles ils avaient eux-mêmes choisi de se soumettre), de l'autre, en contrebande, ils faisaient état dans leurs écrits d'une préoccupation plutôt incongrue mais néanmoins partagée et quasiment obsessionnelle pour le vêtement.

Pendant que dans le monde de la veille se fabriquaient des édifices rationalistes (aussi facile à assembler qu'un avion ou un paquebot) et une nouvelle structure (ou infrastructure), dans le sommeil, un nouveau rêve se mettait en marche et inventait déjà un autre monde qui, en de nombreux points, lui était contradictoire ; un rêve de surface, de façade, d'ornement (ou superstructure), un rêve d'apparence et d'exposition, à la limite un rêve d'extériorité pure. C'est d'ailleurs à ce titre qu'il faut comprendre leur intérêt pour les écrits sur l'art textile et l'ornement de l'architecte et théoricien Gottfried Semper dont ils se sont largement inspirés.

53 C'est là, entre les lignes, que les « images du rêve [...] se dress[ai]ent dans le monde de la veille ${ }^{27}$ » et que s'imposait à l'ordre du jour la «ruse » annonciatrice d'un véritable réveil. 
54 «Ils hésitent encore sur le seuil. Ils s'arrêtent à mi-chemin ${ }^{28}$ " mais, pour les architectes, un seuil est bel et bien élevé puisque se pose désormais la question de le franchir ou de refuser de le faire, de s'arracher au sommeil ou d'y demeurer.

Mais tout hésitants qu'ils sont, ils ont sans doute permis à Benjamin de confirmer qu'un seuil était bel et bien sur le point d'être franchi et de voir, bien que d'une manière différente de celle des surréalistes au même moment, "le champ de ruines que le développement capitaliste des forces productives laisse derrière lui ${ }^{29}$ » et le monde des passages, du fétichisme de la marchandise et de la structure sur le point d'être réellement renversé.

De son côté, parallèlement à son travail sur les passages, Benjamin commence à explorer son propre rêve dont il a pris soin de nous laisser des traces.

Avec ses « protocoles d'expériences faites avec des drogues ", il tente précisément de se plonger artificiellement dans un état de rêve, ou plus exactement dans un état de rêve éveillé, c'est-à-dire dans un état limite, intermédiaire, qui lui permettra d'accéder plus facilement, et de manière presque consciente, à ses propres rêves afin d'en tirer une interprétation dont il espérait sans doute qu'elle rentrerait en résonance avec celle d'un rêve collectif.

Ces expériences, ce choix de protocole, ce processus de questionnement ont été d'autant plus efficaces qu'ils ont été guidés par l'intuition de la réponse car «L'ivresse provoquée par les drogues [explique-t-il] rév[èle] un état plus profond de connaissance ${ }^{30}$ » mais, en même temps, « il vous semble qu'au fond on ne s'engage dans rien trop profondément : qu'on se meut, aussi profondément qu'on pénètre, toujours sur un seuil ${ }^{31} »$.

Pour Benjamin, la prise de drogue a en effet pour caractéristique de faire s'attarder le fumeur sur toutes sortes de seuils. Elle provoque toujours un double effet et le plonge dans une "simultanéité maléfique ${ }^{32}$ ", «la chose et son contraire se présent[ent] [...] avec la même netteté et se concurrenç[ent] [...] jusqu'au supplice ${ }^{33}$ ».

60 Sur le plan de la subjectivité par exemple, la drogue permet une «levée des inhibitions » et l'affirmation d'une souveraineté dont le fumeur n'avait jamais été capable auparavant, mais en même temps, elle fait affleurer à la surface des peurs et des angoisses qui généralement restent enfouies. Comme l'écrit Benjamin ;

cela peut donner même à des natures assez modestes une souveraineté que celles-ci ne possèdent pas à l'origine. [...] Mais, d'autre part, c'est vraisemblablement plus qu'une simple levée des inhibitions qui fait la spécificité de telles réunions [de fumeurs] pour beaucoup de ceux qui ont l'habitude de les organiser. Il semble au contraire que se produise quelque chose comme la rencontre des sentiments d'infériorité, des complexes et des troubles qui ont leur siège dans les différents partenaires. Les drogués s'aspirent pour ainsi dire mutuellement les mauvaises substances de leur existence; nous produisons les uns sur les autres un effet cathartique ${ }^{34}$.

61 Sur le plan social, le «besoin d'être seul et de demeurer avec les autres augmente ${ }^{35}$ » simultanément.

62 Sur le plan de la perception, - et pour en terminer là, «Le fumeur d'opium ou le mangeur de haschich fait l'expérience de la force qu'a le regard d'aspirer cent lieux à un seul endroit ${ }^{36} »$, c'est-à-dire l'expérience d'un regard focalisant, et en même temps, ce regard ne peut être soutenu longtemps de sorte qu'il y a " par instants un éphémère mirage mais il [est] immédiatement dépouillé de son caractère de réalité [...] ${ }^{37} »$. 
Mais, plus important, avec la drogue,

Il y a [...] communication automatique avec soi-même. Des choses prennent la parole sans demander l'autorisation. Ça remonte jusqu'à des sphères très élevées. Il y a un mot de passe silencieux grâce auquel certaines choses franchissent [...] le porche $^{38}$.

Ce mouvement (depuis l'intérieur) rencontre son symétrique (depuis l'extérieur) qu'il explique dans Paris, capitale du XIX siècle: "Notre théorie est que, dans les couches oniriques, la réalité, au lieu d'être, simplement, vient frapper le rêveur. Et je traite des passages exactement comme s'ils étaient au fond quelque chose qui m'était advenu ${ }^{39}$ ".

Avec la drogue, on peut penser que Benjamin cherche à faire coïncider des choses en lui qui prennent la parole sans demander son autorisation avec quelque chose d'autre de la réalité, après les passages, qui viendrait le frapper.

En l'occurrence et entre autres choses, ce qui " franchit le porche ", quand il se met dans cet état de rêve éveillé, ce qui arrive au seuil qui sépare le sommeil de la veille, ce qui lui arrive dans cet état limite c'est, comme par effet de mise en abyme, qu'il développe précisément, dit-il, une "grande sensibilité aux portes ouvertes ${ }^{40}$ », aux fenêtres, aux "rideaux et dentelles ${ }^{41}$ ", aux couleurs, au "monde de surface le plus caché et ordinairement le plus inaccessible que représente l'ornement ${ }^{42}$ ", c'est-à-dire, en termes d'architecture, à tout ce qui constitue le seuil d'un bâtiment, d'une maison, à tout ce qui s'assemble pour constituer le feuilleté complexe de l'épaisseur de la façade séparant un intérieur d'un extérieur, toutes choses que «d'ordinaire [dit-il] nous ne voyons à peine ${ }^{43}$ ».

Lors d'une prise d'opium à Ibiza au printemps 1933 qu'il relate dans Notes sur le crock, il écrit :

[...] prennent place aux côtés de l'ornement certaines autres choses du monde observable le plus banal qui ne transmettent qu'au crock le sens et la signification qui les habitent. En font partie entre autres les rideaux et dentelles. Les rideaux sont les interprètes de la langue du vent. Ils donnent à chaque souffle la forme et la sensualité des formes féminines. Et ils font goûter au fumeur qui se plonge dans leur jeu toute la joie que lui procurerait une danseuse accomplie. Le rideau est-il ajouré, il peut devenir l'instrument d'un jeu encore beaucoup plus étrange. Car ces dentelles se révèlent au fumeur être des sortes de pochoir qu'il plaque sur le paysage pour le métamorphoser de la plus singulière façon. La dentelle assujettit à la mode le paysage, qui transparaît à travers elle, à peu près comme la confection de certains chapeaux assujettit à la mode le plumage d'oiseaux ou la floraison. Il y a de vieilles cartes postales démodées sur lesquelles un "Salut de Bad Ems » divise la ville en promenade thermale, gare, monument de l'empereur Guillaume, école de Karolinenhöhe, chacun disposé dans son petit cercle bien isolé. Des cartes postales de ce genre sont le mieux à même de donner une idée de la façon dont un rideau de dentelle exerce sa domination dans une image de paysage ${ }^{44}$.

68 Valero relate ce même événement du point de vue de Jean Selz, l'ami qui fuma ce jourlà avec lui :

[...] la cérémonie (printemps 1933) des deux fumeurs fut célébrée dans une magnifique chambre de la maison de la rue de la Conquista, située tout en haut de la vieille ville et dont les fenêtres dominaient, par-delà les toits des maisons cubiques et blanches du quartier de la Marina, la petite baie où se tient enfermé le port.

Le regard porté sur l'extérieur par la fenêtre et le paysage contemplé sous les effets de l'opium devinrent les premiers thèmes de réflexion. 
Les toits en terrasse, la courbe du port et les lointaines sierras, enrobés dans les plis du rideau ou capturés par lui, se mouvaient en même temps que lui lorsqu'il était remué - mais à peine - par le peu de vent de cette chaude soirée. La ville et le rideau cessèrent bientôt d'être séparés l'un de l'autre. Et si la ville était devenue tissu, ce tissu devint celui d'un vêtement. Il était notre vêtement, mais s'éloignait de nous. Il fut alors observé que l'opium nous déshabillait du pays où nous vivions. Benjamin ajouta plaisamment que nous faisions de la « rideaulogie $»^{45}$. mailles et dans ses plis, le découpe et l'assujettit au point où rideau et paysage sont pris dans un même mouvement de pli et de dépli et ne font qu'un. vêtement du corps du fumeur, qui, sans lui pour se protéger, a la sensation de devenir nu.

Le paysage vole son rideau au fumeur - comme il lui volerait son vêtement - et choisit à son tour de s'en parer et d'en jouer à la manière d'une danseuse exécutant la danse des voiles, séduisant et laissant le fumeur joyeux mais aussi à découvert et désarmé.

Le rideau n'est pas seulement soumis à la souveraineté du dedans ou du sujet, il est aussi ce par quoi le paysage, se l'appropriant, décide de nous apparaître et de s'imposer à nous.

Dans son ouvrage Sur le haschich Benjamin explique cela autrement :

Il est connu que, lorsqu'on ferme les yeux et qu'on appuie légèrement dessus, apparaissent des figures ornementales sur la forme desquelles on n'a pas d'influence. Les architectures et constellations spatiales qu'on voit devant soi dans le haschich ont à l'origine quelque chose d'apparenté à cela. Quand et sous quelle forme surgissent-elles? C'est de façon d'abord involontaire, tant elles surviennent foudroyantes et impromptues. Puis, une fois qu'elles (sont) là, arrive l'imagination plus consciemment ludique, qui prend certaines libertés avec elles ${ }^{46}$.

Les couleurs et les motifs qui sont capturés par le rideau ne sont plus uniquement un revêtement que le fumeur applique aux formes du paysage à la manière d'un peintre mais ils jaillissent de l'opacité et de la compacité de la matière, vivent par eux-mêmes et viennent jusqu'à lui à travers les mailles du rideau.

De là un " effet incroyablement fort sur le fumeur ", un " effet presque anesthésiant »; Benjamin relate, lors de la même soirée :

ce qui distinguait [les couleurs] m'apparut en ceci qu'elles possédaient avant tout une forme, qu'elles se faisaient parfaitement identiques à la substance où elles apparaissaient. Tandis qu'elles se présentaient tout à fait semblables sur les différentes substances - par exemple un pétale et une feuille de papier--, elles apparaissaient à titre d'intermédiaires ou d'entremetteuses des domaines de la substance ; c'est seulement par elles que les substances les plus éloignées pouvaient fusionner entièrement ${ }^{47}$.

Le rideau n'appartient donc ni au dedans ni au dehors, ni au sujet ni à l'objet; il est l'interface, le plan intermédiaire qu'ils se partagent, ce par quoi, dans un mouvement alternatif et tournant, chambre et ville, dedans et dehors, surface et profondeur, couleur et forme peuvent se mettre en rapport.

La condition pour que cette expérience ait lieu, c'est la suspension et le jeu.

Du fait d'être suspendu et non fondé, le rideau peut occulter la fenêtre et fermer la chambre ou bien au contraire, à la faveur du vent, faire rentrer la ville à l'intérieur ou faire sortir Benjamin dehors, selon une pluralité infinie d'états intermédiaires. 
80 Et comme l'expérience de l'ornement, l'expérience du rideau dans l'ivresse réactive le «sentiment profond de bien-être [...] que nous avons pu observer dans les années d'enfance et dans la fièvre » du fait de leur dimension polysémique. Comme l'ornement, le rideau "se laisse regarder de deux côtés distincts au moins: à savoir comme formation de surface mais aussi comme configuration linéaire [et] la plupart du temps les diverses formes, qui peuvent se regrouper très différemment, autorisent une pluralité de configurations ${ }^{48} »$.

81 C'est donc, au moment même où, entre 1929 et 1934, Benjamin suspend son travail sur Paris, capitale $d u X X^{e}$ siècle et écrit Expérience et pauvreté, qu'il commence à transformer à la fois le rêve du $\mathrm{XIX}^{\mathrm{e}}$ siècle et le cauchemar de la première guerre mondiale comme formes d'intériorité et de ressassement considérables en un nouveau rêve comme forme de seuil et qu'il inaugure un nouveau monde où la rideaulogie remplace les passages et les formes d'expériences précédentes.

82 C'est à cet endroit que Benjamin semble véritablement rencontrer Le Corbusier et l'on peut supposer que l'excitation qu'il éprouve à la lecture de l'ouvrage de Giedion est liée à cette rencontre à distance et à la découverte d'un rêve qu'il n'est plus le seul à faire.

C'est ce qu'il semble confirmer dans une lettre élogieuse adressée à Giedion en 1929, quand il dit de son ouvrage qu'il « est l'un des rares cas, que nous vivons sans doute tous, où avant d'entrer en contact avec quelque chose ou quelqu'un (écrit, maison, homme, etc.), nous savons que ce contact sera hautement significatif, et que ce savoir ne trompe pas. J'apprends dans votre livre (par rapport à tant d'autres, dans la mesure où il me concerne dans le sens le plus immédiat) la différence revivifiante entre la pensée radicale et le savoir radical. Vous possédez ce dernier; par conséquent, vous êtes en mesure d'éclairer la tradition à la lumière du présent - ou plutôt de la découvrir $[\ldots]^{49} \gg$.

84 Dans la dernière partie de l'ouvrage intitulée "Béton armé » - qui est la seule, au moment de l'écriture de sa lettre, que Benjamin ait lue -, Giedion développe en effet une lecture originale de l'architecture moderne.

Si l'aspect industriel de cette architecture - c'est-à-dire le montage d'éléments constructifs préfabriqués et l'emploi de matériaux tels le fer et le béton armé constitue en quelque sorte le fond sur lequel la critique de Giedion s'adosse, ce qui fait singulièrement relief c'est une sémantique renvoyant officieusement à un domaine beaucoup plus archaïque, artisanal et vernaculaire, qui est celui du tissu, ce qui, on peut le parier, n'aura pas manquer d'« électriser » Benjamin.

À propos de Perret d'abord et de son immeuble rue Franklin construit en 1903 à Paris, Giedion écrit : «La façade voit sa planéité détruite. Elle est évidée, elle travaille dans la profondeur, rejaillit vers l'avant, place en saillie six étages en état d'apesanteur, rejette au sixième étage les piliers quadrangulaires. Cette façade a acquis de la mobilité ».

87 Autre bâtiment de Perret, le garage de la rue de Ponthieu à Paris construit en 1905 : «Plus de parois pleines. Ce qui domine ce sont le cadre en béton, dont la fine structure supporte la construction, et les surfaces de verre. Bref, c'est le règne de l'ouverture !»

Enfin, l'église du Raincy (1922-1923) : «La peau extérieure est simplement jetée pardessus [la structure]. Il est logique qu'elle soit percée de partout. [...] Une atmosphère de légèreté, que seule peut procurer la victoire intérieure sur le matériau, emplit cet espace ». 
À propos de « la génération montante » ensuite, et de Le Corbusier en particulier :

Plus qu'aucun autre avant lui, Corbusier a la capacité de faire chanter l'ossature de béton armé que lui a confié la science. [...] Un édifice qui, parce qu'il est suspendu de tout son poids à quelques piliers de béton armé, permet de réduire les murs à une simple peau isolante, voire de les supprimer là où le souhaite le constructeur, possède cette propriété grâce à laquelle Corbusier a créé la maison de l'éternelle ouverture $^{50}$.

90

Il insiste aussi beaucoup sur la notion d'apesanteur en tant que motif essentiel d'arrachement à la tradition : «Pourquoi la maison doit-elle avoir le plus de légèreté possible et être comme en état d'apesanteur? Parce que c'est la seule possibilité de mettre fin à la fatalité d'une monumentalité héritée du passés1 ».

encore, «La performance de Corbusier? Avoir appréhendé la maison avec la sensibilité d'un sismographe et l'avoir libérée de toute sa lourdeur traditionnelle ${ }^{52}$ ».

C'est parce qu'ils sont en train de rêver que les architectes modernes ne peuvent véritablement interpréter ce qu'ils sont en train de construire et que leurs commentateurs tels Giedion ne peuvent décrire ce qu'ils voient en toute conscience (à ce sujet, on peut se rappeler de la célèbre formule de Le Corbusier : "Des yeux qui ne voient pas....53»).

Les uns et les autres recourent nécessairement à des masques ou à des mots qui ne sont pas les leurs - par exemple ceux du xix ${ }^{e}$ siècle - pour dire, mais ce faisant, ils trahissent déjà le rêve d'une nouvelle architecture. S'ils laissent échapper des bribes de ce rêve collectif, ce ne peut être que de manière involontaire, sous la forme de lapsus ou d'actes manqués (leurs écrits).

94 À travers ces silences ou ces masques comme symptôme d'un rêve en train de se faire, comme à travers les mots d'un patient en cure, il ne peut - même s'il en est beaucoup question dans leurs écrits - être question de vérité ou de mensonge, de transparence ou d'opacité délibérées mais seulement d'une sorte de filtre au travers duquel suintent leurs rêves et leur inconscient comme à travers les mailles d'un rideau.

C'est ce que confirmaient Giedion et Benjamin citant Giedion qui, croyant décrire l'expérience esthétique de l'époque par les nouvelles possibilités d'ouverture et l'effacement complet des frontières, en décrivaient en fait et à leur propre insu, une autre.

C'est dans les escaliers battus par le vent de la tour Eiffel ou, mieux encore, entre les poutres métalliques d'un "pont transbordeur» qu'on découvre l'expérience esthétique fondamentale de l'architecture d'aujourd'hui : les choses - les navires, la mer, les immeubles, les mâts, le paysage, le port - passent au travers du mince filet de fer qui demeure tendu dans l'espace. Elles perdent leur forme bien délimitée, elles se mêlent dans un même mouvement tournant vers le bas, elles se confondent toutes en même temps ${ }^{54}$.

Seulement les choses, si elles entrent en contact, ne se confondent pas et, si elles se séparent, elles ne s'excluent pas non plus.

C'est sous le masque de l'ingénieur et par lui que transparaissent les velléités artistiques des architectes modernes. Derrière le masque discursif de l'industrialisation de l'architecture et du progrès technique, derrière le "Nouveau Construire ${ }^{55}$ ", apparaissent une nouvelle esthétique et un nouvel ornement; le blanc de chaux constitue le moyen ultime de ne pas abandonner la décoration, il est sa réaffirmation et non sa disparition. 

permet (système poteau-poutre, poteau-champignon, arc porteur) qu'est en fait rendu possible tout un jeu sur la façade et tout un jeu de la façade qui, se libérant, libère avec elle l'architecture dans son entier.

Cous le masque de la transparence, de l'interpénétration, de la fusion ou de l'unité - « entre intérieur et extérieur les séparations sont tombées ${ }^{56}$ » affirme Giedion - que transparaît la volonté de Le Corbusier de préserver des séparations strictes et de faire de la façade un dispositif de maîtrise du monde.

C'est sous le masque du secret que transparaît la volonté de Loos de ruiner les séparations et de faire de la façade un dispositif de sécrétion du dedans.

Enfin - mais on pourrait continuer - c'est sous le masque du vêtement masculin (costume trois pièces anglais) que transparaît leur goût pour la couleur, le décor, l'accessoire - domaine officiellement circonscrit au féminin.

L'architecture moderne est paradoxale et contradictoire mais, comme les façades historisantes $\mathrm{du} \mathrm{xIX}^{\mathrm{e}}$ siècle, elle a ainsi le mérite de pointer un non vu et c'est, à l'exemple de Benjamin pour les passages, précisément en prélevant dans le rêve collectif des modernes ces aspects les plus contradictoires, qu'il faut tenter une interprétation et en déduire, de la même manière que Benjamin l'avait fait pour le XIXe, une analyse de l'architecture contemporaine afin de déterminer si elle constitue le réveil du rêve collectif du $\mathrm{xx}^{\mathrm{e}}$ siècle ou non.

En tout état de cause, il apparaît que, de façon quasiment inverse au rêve du xixe siècle, l'architecture contemporaine s'attache tout particulièrement à travailler la couche la plus superficielle de ses édifices, à savoir la façade. Pour paraphraser Benjamin, on pourrait dire de l'architecture contemporaine qu'elle élève l'extérieur «à une puissance considérable ».

Comment, dans ces conditions, faut-il alors relire le projet de Benjamin de radicaliser la thèse de Giedion?

Benjamin expose ainsi son projet :

Tentative pour radicaliser la thèse de Giedion. Celui-ci dit que « la construction joue au XIX ${ }^{\mathrm{e}}$ siècle le rôle de l'inconscient ». Ne serait-il pas plus exact de dire qu'elle joue le rôle du processus corporel autour duquel les architectures "artistiques" viennent se poser comme des rêves autour de l'armature du processus physiologique ${ }^{57}$

Faut-il entendre la radicalisation comme une amplification?

C'est, semble-t-il, ce que l'architecture postmoderne, comme l'ont montré Robert Venturi et Denise Scott Brown, a réalisé en repoussant le rêve capitaliste du XIX siècle, la fantasmagorie et le fétichisme de la marchandise en façade et en ne se contentant plus d'assembler des pièces préfabriquées disponibles sur le marché mais en devenant elle-même marchandise, marque ou logo.

Faut-il, comme le suggère Jean-Louis Cohen dans l'avant-propos de Construire en France, construire en fer, construire en béton, entendre la radicalisation comme un renversement qui se serait révélé prémonitoire dans la mesure où les nouvelles façades en seraient aujourd'hui la réalisation concrète? Du rêve collectif du $\mathrm{xIX}^{\mathrm{e}}$ siècle et de sa prolongation au $\mathrm{xx}^{\mathrm{e}}$ siècle, l'architecture contemporaine aurait donc opéré sa révolution copernicienne en retournant comme un gant intérieur et extérieur, rêve et 
conscience et aurait fait du rêve non plus une affaire d'infrastructure mais de superstructure et d'enveloppe.

C'est plutôt en termes de synthèse qu'il nous semble falloir aborder la question de la radicalisation.

Penser à partir de la rideaulogie implique d'appréhender l'expérience du seuil non plus comme un rite de passage (comme l'endormissement ou le réveil) mais plutôt comme une expérience où les "transitions sont devenues de moins en moins perceptibles ${ }^{58}$ " comme c'est le cas dans la psychanalyse.

\section{Benjamin écrit :}

Un des présupposés implicites de la psychanalyse est que l'opposition tranchée du sommeil et de la veille n'a empiriquement aucune valeur pour déterminer la forme de conscience de l'être humain et qu'elle cède la place à une variété infinie d'états de conscience concrets, conditionnés par tous les degrés imaginables de l'état de veille dans les différents centres psychiques ${ }^{59}$.

\section{BIBLIOGRAPHIE}

Benjamin Walter, Sur le haschich et autres écrits sur la drogue, Paris, Christian Bourgois, 1993.

Benjamin Walter, « Le caractère destructif », in Images de pensée, Paris, Christian Bourgois, 1998, p. 173-176.

Benjamin Walter, « De l'espace pour ce qui est précieux », in Images de pensée, Paris, Christian Bourgois, 1998, p. 188-189.

Benjamin Walter, « Expérience et pauvreté », in Euvres II, Paris, Gallimard, 2000, p. 364-372.

Benjamin Walter, « Le surréalisme, le dernier instantané de l'intelligentsia européenne », in Euvres II, Paris, Gallimard (Folio essais), 2000, p. 113-134.

Benjamin Walter, Paris, capitale du XIX ${ }^{e}$ siècle. Le livre des Passages, Paris, Éditions du Cerf, 2006.

Giedion Sigfried, Construire en France, construire en fer, construire en béton, Paris, Éd. de la Villette, 2000.

Le Corbusier, Vers une architecture, Paris, Arthaud, 1977.

Soulillou Jacques, Le livre de l'ornement et de la guerre, Marseille, Éd. Parenthèses, 2003.

Valero Vicente, Expérience et pauvreté, Walter Benjamin à Ibiza (1932-1933), Juan Vila (trad.), Rodez, Le Rouergue/Chambon, 2003.

\section{NOTES}

1. Walter Benjamin, Paris, capitale du XIX $X^{e}$ siècle. Le livre des Passages, Paris, Éditions du Cerf, 2006,

p. 424-425.

2. Ibid., p. 424.

3. Ibid., p. 405-406. 
4. Ibid., p. 893.

5. Ibid., p. 46.

6. Ibid., p. 425.

7. Walter Benjamin, « Expérience et pauvreté », in Euvres II, Paris, Gallimard, 2000, p. 365-366.

8. Ibid., p. 368.

9. Ibid., p. 369.

10. Walter Benjamin, «Le surréalisme, le dernier instantané de l'intelligentsia européenne », in Euvres II, Paris, Gallimard (Folio essais), 2000, p. 118.

11. Walter Benjamin, «Le caractère destructif », in Images de pensée, Paris, Christian Bourgois, 1998, p. 173-175.

12. Vicente Valero, Expérience et pauvreté, Walter Benjamin à Ibiza (1932-1933), Juan Vila (trad.), Rodez, Le Rouergue/Chambon, 2003, p. 45.

13. Ibid., p. 25-26.

14. Walter Benjamin, « De l'espace pour ce qui est précieux », in Images de pensée, Paris, Christian Bourgois, 1998, p. 189.

15. Vicente Valero, Expérience et pauvreté, Walter Benjamin à Ibiza (1932-1933), p. 24.

16. Walter Benjamin, «Expérience et pauvreté », p. 372.

17. Walter Benjamin, Paris, capitale du XIX ${ }^{e}$ siècle, p. 192.

18. Ibid., p. 881.

19. Ibid., p. 410.

20. Jacques Soulillou, Le livre de l'ornement et de la guerre, Marseille, Éd. Parenthèses, 2003, p. 27.

21. Sigfried Giedion, Construire en France, construire en fer, construire en béton, Paris, Éd. de la Villette, 2000, p. 85.

22. Ibid., p. 99.

23. Walter Benjamin, Paris, capitale du XIX siècle, p. 482.

24. Ibid., p. 894.

25. Walter Benjamin, « Expérience et pauvreté », p. 371.

26. Walter Benjamin, Paris, capitale du XIX siècle, p. 405-406.

27. Ibid., p. 894.

28. Ibid., p. 913.

29. Ibid., p. 913.

30. Vicente Valero, Expérience et pauvreté, Walter Benjamin à Ibiza (1932-1933), p. 106.

31. Walter Benjamin, Sur le haschich et autres écrits sur la drogue, Paris, Christian Bourgois, 1993, p. 10.

32. Ibid., p. 16.

33. Ibid., p. 39.

34. Ibid., p. 86-87.

35. Ibid., p. 16.

36. Ibid., p. 86.

37. Ibid., p. 36.

38. Ibid., p. 22.

39. Walter Benjamin, Paris, capitale du XIXe siècle, p. 893.

40. Walter Benjamin, Sur le haschich et autres écrits sur la drogue, p. 11.

41. Ibid., p. 83.

42. Ibid., p. 82.

43. Ibid., p. 82.

44. Ibid., p. 83-84.

45. Vicente Valero, Expérience et pauvreté, Walter Benjamin à Ibiza (1932-1933), p. 107-108.

46. Walter Benjamin, Sur le haschich et autres écrits sur la drogue, p. 16. 
47. Ibid., p. 84-85.

48. Ibid., p. 82.

49. Sigfried Giedion, Construire en France, construire en fer, construire en béton, p. 17

50. Ibid., p. 84.

51. Ibid., p. 85.

52. Ibid., p. 100.

53. Le Corbusier, Vers une architecture, Paris, Arthaud, 1977, p. 65.

54. Walter Benjamin, Paris, capitale du XIX ${ }^{e}$ siècle, p. 475.

55. Sigfried Giedion, Construire en France, construire en fer, construire en béton, p. 114.

56. Ibid., p. 85.

57. Walter Benjamin, Paris, capitale du XIX $X^{e}$ siècle, p. 408.

58. Ibid., p. 512-513.

59. Ibid., p. 406-407.

\section{RÉSUMÉS}

Pour Benjamin, s'imposait à son époque une double tâche : il s'agissait d'une part, de sortir du cauchemar de la première guerre mondiale et, d'autre part, de sortir du rêve du xixe siècle, dont, du fait même de cette guerre, il n'avait pas été possible de se réveiller. C'est dans l'architecture moderne - particulièrement chez Le Corbusier - qu'il pensait avoir trouvé les preuves d'un renversement dialectique ou révolutionnaire. Seulement, on peut penser qu'elle a plutôt constitué une "fausse délivrance» dont la violence aura masqué un nouveau rêve collectif pourtant déjà en train de se faire.

INDEX

Mots-clés : rideaulogie, maison de rêve, seuil 